\title{
CR Research Square \\ Aerobic Vaginitis in the Third Trimester and Its Impact on Pregnancy Outcomes
}

\section{Anh Thi Chau Nguyen}

Hue University of Medicine and Pharmacy, Hue University

\section{Na Thi Le Nguyen}

Becamex International Hospital

\section{Thu Thi Anh Hoang}

Hue University of Medicine and Pharmacy, Hue University

\section{Tuyen Thi Nguyen}

Hue University of Medicine and Pharmacy, Hue University

\section{Trang Thi Quynh Tran}

Hue University of Medicine and Pharmacy, Hue University

\section{Dan Nu Tam Tran}

Hue University of Medicine and Pharmacy, Hue University

\section{Linh Manh Tran}

Hue University of Medicine and Pharmacy, Hue University

\section{Duc Huu Chau Nguyen}

Hue University of Medicine and Pharmacy, Hue University

\section{Anh Thi Kim Nguyen}

Hue University of Medicine and Pharmacy, Hue University

\section{Tam Minh Le}

Hue University of Medicine and Pharmacy, Hue University

\section{Binh Duy Ho}

Hue University of Medicine and Pharmacy, Hue University

\section{Tiiu Rööp}

University of Tartu

\section{Siiri Kõljalg}

University of Tartu

Jelena Štšepetova

University of Tartu

\section{An Van Le}

Hue University of Medicine and Pharmacy, Hue University Andres Salumets

Competence Centre on Health Technologies AS

Reet Mändar ( $\nabla$ reet.mandar@ut.ee ) 


\section{Research Article}

Keywords: aerobic vaginitis, pregnancy, puerperal sepsis, neonatal infections

Posted Date: January 20th, 2022

DOI: https://doi.org/10.21203/rs.3.rs-1208688/v1

License: (c) (i) This work is licensed under a Creative Commons Attribution 4.0 International License. Read Full License 


\section{Abstract \\ Background}

Aerobic vaginitis (AV) is a vaginal inflammation characterized by disruption of the lactobacillus microbiota and increased counts of different aerobic bacteria. AV may result in severe complications, especially during pregnancy, including preterm delivery, neonatal and maternal infections. This study aimed to determine the prevalence of $A V$ in the third trimester of pregnancy, and the relationship between AV and pregnancy outcomes.

\section{Methods}

A cross-sectional descriptive study included 323 pregnant women attending for routine antenatal care in the Hue University Hospital. Vaginal samples collected at the third trimester of pregnancy were evaluated for AV according to the scoring system of Donders and cultured for identification of predominant bacteria. Pregnancy was followed to its end, and pregnancy outcomes were recorded for both mothers and infants.

\section{Results}

The proportion of pregnant women diagnosed with $\mathrm{AV}$ in the third trimester was found to be $15.5 \%$, with the vast majority of the cases (84\%) displaying the light $\mathrm{AV}$ and $16 \%$ the moderate $\mathrm{AV}$. The vaginal cultures in the women with AV revealed most frequently Streptococcus agalactiae (6\%), followed by Enterococcus spp (4\%), Staphylococcus aureus (4\%), and Acinetobacter baumannii (2\%). In addition, AV during the last trimester of pregnancy was associated with an increased risk of puerperal sepsis (OR 8.65, $95 \% \mathrm{Cl}: 1.41-53.16, \mathrm{p}=0.020)$ and there was a slightly increased risk for neonatal infections, which was statistically insignificant.

\section{Conclusions}

The proportion of AV is relatively high in Vietnamese pregnant women. Since it is associated with an increased risk of puerperal sepsis, it needs to be diagnosed and treated before delivery.

\section{Background}

In 2002, the term "aerobic vaginitis" (AV) was first identified by Donders et al. [1]. AV is described as a depletion of the Lactobacillus microbiota and an increase in aerobic bacteria derived mainly from the gastrointestinal tract, accompanied by inflammatory markers and deficient epithelial maturation (Table 1). $A V$ is characterized by the presence of several aerobic and facultative bacteria, such as Streptococcus agalactiae, Enterococcus faecalis, Escherichia coli, coagulase negative staphylococci (CoNS) and 
Staphylococcus aureus [2]. Clinical presentation includes purulent homogenous discharge (yellow or yellow-green), foul-smelling rotten odor, introital and vaginal redness (in case of severe AV, also ecchymotic bleeding points and erosions), stinging and burning sensations, and dyspareunia. Symptoms can be present for a long period of time and fluctuate in terms of severity [3].

Table 1. Criteria for the microscopic diagnosis of aerobic vaginitis (AV) by Donders et al [1]

\begin{tabular}{|c|c|c|c|c|c|}
\hline $\begin{array}{l}\text { AV } \\
\text { score }\end{array}$ & $\begin{array}{l}\text { Lactobacillary grades } \\
\text { (LBG) }\end{array}$ & $\begin{array}{l}\text { Number of } \\
\text { leukocytes }\end{array}$ & $\begin{array}{l}\text { Proportion } \\
\text { of toxic } \\
\text { leukocytes\# }\end{array}$ & $\begin{array}{l}\text { Background } \\
\text { flora }\end{array}$ & $\begin{array}{l}\text { Proportion of } \\
\text { parabasal } \\
\text { epitheliocytes* }\end{array}$ \\
\hline 0 & $\begin{array}{l}\text { I and Ila } \\
\text { LBG I - numerous } \\
\text { pleomorphic LB, no } \\
\text { other bacteria } \\
\text { LBG Ila - mixed flora, } \\
\text { but predominantly LB }\end{array}$ & $\leq 10 / \mathrm{hpf}$ & $\begin{array}{l}\text { None or } \\
\text { sporadic }\end{array}$ & $\begin{array}{l}\text { Unremarkable } \\
\text { or cytolysis }\end{array}$ & $<1 \%$ \\
\hline 1 & $\begin{array}{l}\text { Ilb } \\
\text { Mixed flora, but } \\
\text { proportion of LB } \\
\text { severely decreased due } \\
\text { to increased number of } \\
\text { other bacteria }\end{array}$ & $\begin{array}{l}>10 / \mathrm{hpf} \text { and } \\
\leq 10 / \text { epithelial } \\
\text { cell }\end{array}$ & $\begin{array}{l}\leq 50 \% \text { of } \\
\text { leukocytes }\end{array}$ & $\begin{array}{l}\text { Small } \\
\text { coliform } \\
\text { bacilli }\end{array}$ & $<10 \%$ \\
\hline 2 & $\begin{array}{l}\text { III } \\
\text { LB severely depressed } \\
\text { or absent because of } \\
\text { overgrowth of other } \\
\text { bacteria }\end{array}$ & $\begin{array}{l}>10 / \\
\text { epithelial cell }\end{array}$ & $\begin{array}{l}>50 \% \text { of } \\
\text { leukocytes }\end{array}$ & $\begin{array}{l}\text { Cocci or } \\
\text { chains }\end{array}$ & $\geq 10 \%$ \\
\hline
\end{tabular}

Hpf - high-power field (400x magnification); LB - lactobacilli; LBG - lactobacillary grade.

* Parabasal epitheliocytes - small round immature epithelial cells with a high nuclear to cytoplasmic ratio.

\# Toxic leucocytes - swollen leucocytes containing granules showing lysosomal activity.

Total score of $<3$, no signs of AV; score 3-4, light AV; score 5-6, moderate AV; score 7-10, severe AV (the latter group resembles 'desquamative atrophic vaginitis').

AV has been observed in approximately $4-8 \%$ of pregnant women [3] and in $5-24 \%$ of women complaining of abnormal vaginal discharge [2]. To date, AV has not yet been considered a specific concern for pregnancy. It is often mistakenly diagnosed with bacterial vaginosis (BV), an anaerobic imbalance of vaginal microbiota. Meanwhile, between AV and BV, there are numerous distinctive characteristics of clinical symptoms like type of vaginal discharge, Whiff test results, presence of 
inflammation, level of immune reaction and different cellular pattern. Diagnostic confusion can lead to treatment disappointment and has been related to serious pregnancy complications such as preterm birth, premature rupture of membranes (PROM), miscarriage, chorioamnionitis and neonatal infections [3-6]. At the same time, preterm birth and neonatal infection are two of the top three causes of neonatal mortality (along with neonatal asphyxia). Less is known about the association of AV with puerperal sepsis, though this is one of the top five causes of maternal deaths worldwide.

Screening for vaginitis during pregnancy is prescribed routinely at the time of 12-16 weeks of pregnancy in most countries. However, in Vietnam, there is no scheduled testing for pregnant women, especially those who do not have symptoms that indicate infection. This may lead to the above-mentioned obstetric risks and increase the rate of admission to the neonatal intensive care unit (NICU). For the above reasons, we carried out the study in order to reveal the proportion of women having AV during the last trimester of pregnancy. In addition, we also aimed to look for the relationship between AV and pregnancy outcomes.

\section{Methods}

\section{Study subjects}

Participants for this cross-sectional descriptive study were recruited among pregnant women who attended routine antenatal care in the Hue University Hospital, Vietnam, from July 2018 until January 2019. All the women had live fetuses without fetal anomalies. The exclusion criteria were previously diagnosed rupture of membranes, antepartum hemorrhage, vaginal douching before or during vaginal specimen collection; those who had been treated for reproductive tract infections or who had used antibiotics for some other reasons within the last week.

The sample size was calculated according to the prevalence of pregnant women in the last trimester carrying microorganisms that cause lower genital tract diseases, according to the previous research from the Hue University Hospital (42.9\%) [7]. With $95 \% \mathrm{Cl}$ and 0.16 of relative deviation, our study needed the minimum sample size of 200 cases.

This study was approved by the Ethics Committee of Hue University of Medicine and Pharmacy, Vietnam (No. H2018/162 dated May 24th, 2018). All study participants accepted and signed the consent form. Participation in the study was voluntary. Written informed consent was obtained from all study subject. All methods were performed in accordance with the relevant guidelines and regulations, including Declaration of Helsinki.

\section{Clinical investigations and sample collection}

Pregnant women in the last trimester of pregnancy ( $\geq 28$ weeks) eligible for treatment and/or labor at the Hue University Hospital were asked for general information, clinical examination and sampling. Background data included age, education, profession, marital status, and history of reproductive health (genital tract infections, abortions, and miscarriages). 
The women were monitored until delivery, and both maternal and neonatal outcomes were recorded. Clinical data of the pregnant women included PROM, preterm premature rupture of membranes (pPROM), chorioamnionitis, delivery mode, and puerperal health. Clinical data of the newborns included birth weight, Apgar scores, and neonatal infections.

Vaginal samples were collected mostly (91\%) after 37 weeks of pregnancy. Vaginal fluid was taken from the vagina, at the site of suspected infection or at the posterior vaginal fornix. Two sterile cotton swabs were used for sampling. The swabs were transported to the Department of Microbiology in the Hue University Hospital of Medicine and Pharmacy within $2 \mathrm{~h}$ or stored at $4^{\circ} \mathrm{C}$ within $12 \mathrm{~h}$ before sending to the lab. At the lab, one swab was used for making Gram stained slide smear, the second swab was frozen in cryotube (with $\mathrm{BHI}$ broth and $20 \%$ glycerol) for the subsequent cultures in University of Tartu, Estonia.

\section{Laboratory methods}

The microscopic AV diagnostic criteria proposed by Donders et al were applied under microscope applying both $400 x$ and $1000 x$ magnification $[3,8,9]$. Lactobacillary grades, other bacteria, leukocytes and parabasal epithelial cells were recorded. AV was diagnosed as indicated in Table 1. AV was classified as light AV (score 3 or 4), moderate AV (score 5 or 6 ) and severe AV (score $\geq 7$ ).

In addition, vaginal fluid was cultured onto blood agar media, the cultures were incubated for $18-24 \mathrm{~h}$ in aerobic conditions. The predominant colonies were counted and identified by MALDI-TOF MS (Bruker Daltonics) at the Department of Microbiology in University of Tartu, Estonia.

\section{Statistical methods}

The collected data were stored in MS Excel 2016. SPSS 20.0 software was used for analysis and processing the data. Factors related to AV were determined to have statistically significant differences if $p$ $<0.05$.

\section{Results}

\section{Clinical characteristics of the study subjects}

In total 323 cases were eligible to be enrolled in the study (Table 2). Their mean age was $28.3 \pm 5.1$ years, $64.1 \%$ of them belonged to age group 25-34 years. Most of the women had education at the secondary school level and above. In term of career, public servants and housewives accounted for the majority. Vast majority of women were married, while only $0.6 \%$ of respondents were single. The proportion of pregnant women with a history of lower genital tract infections accounted for $18.9 \%$, and that of with previous abortion or miscarriage $20.7 \%$. 
Table 2

Background characteristics of the study group

\begin{tabular}{|c|c|c|}
\hline Variable & $\mathbf{N}$ & Rate (\%) \\
\hline \multicolumn{3}{|l|}{ Age (years) } \\
\hline$-<18$ & 3 & 0.9 \\
\hline$-18-24$ & 75 & 23.2 \\
\hline$-25-34$ & 207 & 64.1 \\
\hline$-\geq 35$ & 38 & 11.8 \\
\hline Average age (years) & \multicolumn{2}{|c|}{$28.3 \pm 5.1$} \\
\hline \multicolumn{3}{|l|}{ Profession } \\
\hline - Public servants & 133 & 41.2 \\
\hline - Agriculture-forestry-fishery & 13 & 4.0 \\
\hline - Housewife & 92 & 28.5 \\
\hline - Business & 50 & 15.5 \\
\hline - Other & 35 & 10.8 \\
\hline \multicolumn{3}{|l|}{ Education level } \\
\hline - Illiteracy & 0 & 0.0 \\
\hline - Primary school & 9 & 2.8 \\
\hline - Secondary or high school & 222 & 68.7 \\
\hline - College or above & 92 & 28.5 \\
\hline \multicolumn{3}{|l|}{ Marital status } \\
\hline - Married & 321 & 99.4 \\
\hline - Unmarried & 2 & 0.6 \\
\hline \multicolumn{3}{|l|}{ Sampling time (week of pregnancy) } \\
\hline$-<37$ & 30 & 9.3 \\
\hline$-\geq 37$ & 293 & 90.7 \\
\hline History of lower genital tract infections & 61 & 18.9 \\
\hline History of abortion or miscarriage & 67 & 20.7 \\
\hline
\end{tabular}




\section{Prevalence and impact of aerobic vaginitis}

The prevalence of $\mathrm{AV}$ in Vietnamese pregnant women made up 15.5\%, most of these women (42 out of 50 ) had light AV and 8 women had moderate AV (Table 3). The cultures revealed different aerobic and facultative bacteria in these women, most frequently Streptococcus agalactiae (6\%), Enterococcus spp (4\%) and Staphylococcus aureus (4\%), followed by Acinetobacter baumannii (2\%) (Table 4). The cultures in the women without $\mathrm{AV}$ revealed most frequently enterococci $(6.7 \%)$

Table 3

AV status in the investigated pregnant women

\begin{tabular}{|lll|}
\hline AV status & N & Rate (\%) \\
\hline No signs of AV & 273 & 84.5 \\
\hline AV & & \\
\hline - Light (score 3-4) & 42 & 13.0 \\
\hline - Moderate (score 5-6) & 8 & 2.5 \\
\hline - Severe (score 7-10) & 0 & 0 \\
\hline Total & $\mathbf{3 2 3}$ & $\mathbf{1 0 0}$ \\
\hline
\end{tabular}

Table 4

Results of the vaginal cultures in the pregnant women having $\mathrm{AV}$

\begin{tabular}{|lll|}
\hline Culture result of vaginal fluid & N & Rate (\%) \\
\hline Negative & 42 & 84.0 \\
\hline Positive & & \\
\hline - Acinetobacter baumannii & 1 & 2.0 \\
\hline - Enterococcus spp & 2 & 4.0 \\
\hline - Staphylococcus aureus & 2 & 4.0 \\
\hline - Streptococcus agalactiae & 3 & 6.0 \\
\hline Total & $\mathbf{5 0}$ & $\mathbf{1 0 0}$ \\
\hline
\end{tabular}

AV was associated with an increased risk of postpartum infection, the puerperal sepsis (OR $8.65,96 \% \mathrm{Cl}$ : 1.4-53.2, $p=0.020$ ) (Table 5). Slightly increased risk for neonatal infections was also seen, but this difference (16.0\% for AV vs. $10.3 \%$ for non-AV pregnancies) was not statistically significant. 
Association of pregnancy outcome with AV

\begin{tabular}{|c|c|c|c|c|}
\hline \multirow[t]{2}{*}{ Variable } & AV present $(n=50)$ & No AV $(n=273)$ & \multirow[t]{2}{*}{ OR $(95 \% \mathrm{Cl})$} & \multirow[t]{2}{*}{$\mathbf{p}$} \\
\hline & $\mathbf{N}(\%)$ & $\mathbf{N}(\%)$ & & \\
\hline Preterm labor & $1(2.0)$ & $9(3.3)$ & $0.60(0.07-4.83)$ & 0.631 \\
\hline \multicolumn{5}{|l|}{ Rupture of membranes: } \\
\hline - PROM * & $15(30.0)$ & $91(33.3)$ & $0.86(0.45-1.65)$ & 0.645 \\
\hline - pPROM * & $0(0.0)$ & $2(0.7)$ & $0.68(0.03-14.46)$ & 0.807 \\
\hline Chorioamnionitis & $0(0.0)$ & $0(0.0)$ & - & - \\
\hline \multicolumn{5}{|l|}{ Childbirth: } \\
\hline - Caesarean section & $26(52.0)$ & $152(55.7)$ & $0.86(0.47-1.58)$ & 0.631 \\
\hline - Vaginal labor & $24(48.0)$ & $121(44.3)$ & - & - \\
\hline Puerperal sepsis & $3(6.0)$ & $2(0.7)$ & $8.65(1.41-53.16)$ & 0.020 \\
\hline Low birth weight $(<2500 \mathrm{~g})$ & $1(2.0)$ & $11(4.0)$ & $0.49(0.06-3.85)$ & 0.495 \\
\hline \multicolumn{5}{|l|}{ Apgar score: } \\
\hline - Apgar score 1 minute $<8$ & $0(0.0)$ & $2(0.7)$ & $1.08(0.05-22.73)$ & 0.963 \\
\hline - Apgar score 5 minute $<8$ & $0(0.0)$ & $1(0.4)$ & $1.80(0.07-44.78)$ & 0.720 \\
\hline Neonatal infections & $8(16.0)$ & $28(10.3)$ & $1.71(0.73-4.00)$ & 0.219 \\
\hline \multicolumn{5}{|c|}{ PROM - premature rupture of membranes; $\mathrm{PPROM}$ - preterm premature rupture of membranes } \\
\hline
\end{tabular}

\section{Discussion}

Our data showed that $15.5 \%$ of Vietnamese pregnant women had AV in the third trimester of pregnancy, and majority of these cases represented AV light form (13\%), while the moderate AV was diagnosed only in $2.5 \%$ of pregnant women. However, despite of the light/moderate form, the AV was significantly associated with an increased risk of postpartum infections that is considered to be one of the leading causes of maternal death worldwide.

\section{Etiopathogenesis, diagnosis and treatment of AV}

Healthy vaginal microbiota is dominated by lactobacilli while imbalance of this community may result in $\mathrm{AV}, \mathrm{BV}$ or candidiasis. In case of $\mathrm{AV}$, lactobacilli counts are decreased while that of several enteric bacteria are increased, including S. agalactiae, E. faecalis, E. coli, CoNS and S. aureus [2]. During last years, some 
studies have applied next-generation sequencing to AV patients. These studies have indicated increased number of species [10], elevated proportions of Actinobacteria and Bacteroidetes [11], and surprisingly also high abundance of Gardnerella vaginalis and Prevotella bivia, in addition to that of S. agalactiae [8]. Not only the species composition, but also their microbial concentration may be of importance. Moreover, it is not finally clear whether this imbalanced community is of the causative factor of the AV or the associated feature present in AV. It has also been proposed that AV may be an immunological disorder with an influence on the vaginal microbiota [12]. This idea is supported by variable degrees of inflammation, not only measured by the number of leucocytes, but also by the proportions of so-called 'toxic leucocytes' (swollen leucocytes containing granules showing lysosomal activity), imbalance of other immune markers and a variable presence of parabasal or immature epithelial cells. Other possible pathogenetic factors include low local estrogen level, presence of sialidase-producing bacteria and deficiency of vitamin D [3], while also presence of some sexually transmitted infections [4] has also been linked to AV. Intrauterine devices, being unmarried, long term use of antibiotics and frequent vaginal douching have been considered to contribute to the AV etiology [13].

Diagnosis of $\mathrm{AV}$ can be made using wet mount phase contrast microscopy. An AV score is calculated according to lactobacilli grade (LBG), presence of inflammation, proportion of toxic leukocytes, microbiota characteristics, and parabasal epithelial cells. A score ranging from 0 to 2 is assigned to each of the above-mentioned characteristics, and $\mathrm{AV}$ is diagnosed according to the composite score: 3-4 indicates light AV, 5-6 represents moderate AV and 7-10 represents severe AV (Table 1). Cultures can give additional information about the microbiota composition but the diagnosis cannot rely on the cultures only. To circumvent the hurdle of microscopic investigation, some attempts have been made to develop nucleic-acid-based and enzymatic diagnostic tests, but the detailed information obtained with microscopy, especially cellular pattern, has remained irreplaceable $[3,14]$. The AV diagnostic criteria among previous studies have not been unanimous. Choice of diagnostic approach depends on the suspected diagnosis and the readiness of the health care system to recognize, diagnose and treat AV. Clinical picture is often nonspecific, affecting the initial diagnostic approach as well as prescribing inaccurate medication. Most clinicians deem that the expected AV rate may be higher and that the majority of $\mathrm{AV}$ cases remain undiagnosed. Though, many researchers and clinicians increasingly focus on $\mathrm{AV}$ in case of vaginitis symptoms. Majority of studies have used Gram stain microscopy to reveal lactobacilli and other bacteria, rate of leukocytes and presence of parabasal epitheliocytes $[15,16]$. We have applied similar diagnostic method on the group of Vietnamese pregnant women. In addition to microscopy, we performed also cultures that revealed common list of bacteria that is characteristic to AV. S. agalactiae, enterococci and $S$. aureus were the three predominant bacteria in our AV patients being somewhat different from the previous studies [6, 9], where E. coli made up $80-90 \%$ of AV cases in pregnant women. To date, there is not much information on the consistency between AV diagnostic criteria by microscopy and culture, thus explaining the discrepancies between the results of the current and previous studies.

One component of the AV-complex, the group B streptococci (GBS) or S. agalactiae is a common causative agent of neonatal infections, therefore routine screening of this species at the end of 
pregnancy has been recommended [17-19]. Its prevalence is about 10-30\% being fairly higher than the reported AV during pregnancy in the present study. It must be pointed that the presence of this species does not mean AV diagnosis. Our previous study revealed $6 \%$ and $9.5 \%$ when a culture or SYBR Green real-time PCR technique was applied to 116 pregnant women to detect GBS, respectively [20]. In the present study, culture revealed this species from 10 women (3\%) from the 323 cohort of pregnant women.

The best treatment for AV patients is not yet fully determined, but it must be tailored according to the microscopic findings and the patient's needs. There is a role for estrogen therapy, corticosteroids, local antiseptics, antibiotics (clindamycin, moxifloxacin, carbapenems, combinations of beta-lactam with betalactamase inhibitor and others) and probiotics, depending on the leading symptoms and comorbidity. Metronidazole that is commonly used to treat BV is not effective in case of AV [3]. It cannot be forgotten that $A V$ can co-occur with other genital tract infections, such as BV, candidiasis and sexually transmitted infections, therefore these diseases need to be ruled out in AV patients.

\section{Prevalence of $\mathrm{AV}$ in pregnancy}

The prevalence of $\mathrm{AV}$ is largely unknown, both in pregnant and unpregnant women, since routine diagnostics in case of vaginitis includes detection of BV, candidiasis and trichomoniasis but not AV. Since reliable tests are not commercially available and wet mounts are not routinely performed in many settings, the AV is likely underreported. Therefore, it has been recommended to include AV in the diagnostic workup for patients seeking medical care for vaginitis symptoms [10].

In our study, the prevalence of $A V$ in late pregnancy was $15.5 \%$, mainly representing the light form of the disease $(13.0 \%)$ and lesser extent $(2.5 \%)$ the moderate form of AV. No severe cases were found in our study. These numbers are generally comparable to the previous reports where the prevalence of AV has been reported to be $4-8 \%$ in pregnant women and $7-13 \%$ in non-pregnant women [3]. Hence, the AV is less common than $\mathrm{BV}$, and it tends to be slightly less represented among pregnant than non-pregnant women, being probably associated with physiological changes in the vaginal environment. However, according to some studies the AV in pregnant women may be even higher in prevalence than in nonpregnant women [21]. Still, the available data is not yet sufficient to draw the final conclusions, and moreover, the used methods have varied between the studies.

\section{Impact of AV on pregnancy outcome}

Previous studies have revealed that $\mathrm{AV}$ can be related to different pregnancy complications like chorioamnionitis, fetal infection, premature birth, PROM and PPROM as well as neonatal infections [3, 4], less data is available about postpartum infections [4]. Thus, the profile of complications tends to be quite similar to that of BV.

In our study, $6 \%$ of pregnant women diagnosed with AV had a puerperal sepsis, whereas in the group without $A V$, the rate was only $0.7 \%$. Thus, the $A V$ patients had almost nine-fold increased risk $(O R=8.65)$ of postpartum infection compared to the controls. Postpartum infections account for a significant, and often preventable, portion of the global healthcare burden. They are one of the top five causes of 
maternal deaths worldwide and account for $10-15 \%$ of deaths in the postpartum period. Puerperal sepsis causes more than 75,000 maternal deaths annually [22]. Abnormal vaginal microbiota forms an important reservoir for causative agents of these infections. This is a strong indication that AV needs to be diagnosed and treated in pregnant women. The reasons why AV can be associated with different neonatal and maternal adverse pregnancy outcomes are not finally known but the bacterial pattern and load may likely have its impact.

The most prevalent bacteria in our AV patients were $S$. agalactiae, enterococci and $S$. aureus, which may cause puerperal and neonatal infections. The frequency of other pregnancy complications and caesarean section was not different in women with and without $A V$ in our study, and the preterm birth rate was low both groups ( $2 \%$ vs $3.3 \%$ ). Yet, to the contrary of the current study, some previous studies have revealed association of AV with preterm labor and PROM [3, 4]. In our research, we analyzed the rate of amniotic fluid rupture at all the gestational ages, without seeing the effect of AV on either PROM or PPROM.

Even though screening and prevention policies for $S$. agalactiae have been systematically introduced, early neonatal infection caused by this species continues to be a problem. However, since the turn of the millennium, the neonatal infections have remained on the plateau [19]. The screening and prevention of neonatal Group B streptococcus infections have currently been primarily successfully applied in term infants, while the current pattern of neonatal infection and mortality caused by $S$. agalactiae has still remained the serious risk factor for preterm neonates [17]. In our study, both groups had similar preterm birth rates, but prevalence of $S$. agalactiae was only $3 \%$ in the full cohort, being lower than in the general reports. In the current study, the neonatal sepsis was found in 36 neonates $(11.1 \%$ out of all 323 neonates), while the neonatal sepsis occurred slightly, but insignificantly more frequently in AV (16\%) than in non-AV (10.3\%) groups of pregnant women. Still, we have no data and solid clue about the etiology of the neonatal infections in our study groups.

\section{Conclusions}

The proportion of Vietnamese pregnant women diagnosed with $\mathrm{AV}$ in the third trimester accounts for $15.5 \%$ of all pregnant women, with the vast majority of cases representing the light disease form. Pattern of cultured bacteria includes common intestinal aerobes with $S$. agalactiae, Enterococcus sp and $S$. aureus being the most prevalent. AV in pregnant women is significantly associated with an increased risk of postpartum infections and therefore needs to be diagnosed and treated before delivery.

\section{Abbreviations}

AV

aerobic vaginitis

BV

bacterial vaginosis

CoNS

Page 12/16 
coagulase negative staphylococci

GBS

group B streptococci

LBG

lactobacilli grade

NICU

neonatal intensive care unit

PROM

premature rupture of membranes

pPROM

preterm premature rupture of membranes.

\section{Declarations}

\section{Ethics approval and consent to participate}

This study was approved by the Ethics Committee of Hue University of Medicine and Pharmacy, Vietnam (No. H2018/162 dated May 24th, 2018). All study participants accepted and signed the consent form. Participation in the study was voluntary. Written informed consent was obtained from all study subject. All methods were performed in accordance with the relevant guidelines and regulations, including Declaration of Helsinki.

\section{Consent for publication}

Not applicable.

\section{Availability of data and materials}

The datasets used and analysed during the current study are available from the corresponding author on reasonable request.

\section{Competing interests}

None.

\section{Funding}

This study was supported by Hue University of Medicine and Pharmacy, Estonian Research Council (grant No. IUT34-19 and PRG1076), Estonian Ministry of Education and Research (grant No. KOGU-HUMB) and Horizon 2020 innovation (ERIN, grant no. EU952516) of the European Commission. The funders had no role in the design of the study and collection, analysis, and interpretation of data and in writing the manuscript.

\section{Authors' contributions}


All authors have read and approved the final manuscript. The specific contributions of each author to the final manuscript were as follows: ATCN - study design, collecting of specimens, testing of specimens, performing mass-spectrometry analyses, data management, manuscript preparation; NTLN - collecting of specimens, assisting with analysis of datasets, manuscript preparation; TTAH - assisted with analysis of datasets, manuscript preparation; TTN - testing of specimens; TTQT - analysis of datasets, manuscript preparation; DNTT - analysis of datasets; ATKN - analysis of datasets; LMT - manuscript preparation; $\mathrm{DHCN}$ - analysis of datasets, manuscript preparation; TML - manuscript preparation; BDH - manuscript preparation; TR - testing of specimens, performing mass-spectrometry analyses, manuscript preparation; SK - testing of specimens, manuscript preparation; JŠ - testing of specimens, manuscript preparation; ALV - data review, manuscript preparation, supervision; AS - study design, manuscript preparation, supervision; RM - study design, data review, manuscript preparation, supervision.

\section{Acknowledgements}

None.

\section{Authors' information}

${ }^{1}$ Department of Microbiology, Hue University of Medicine and Pharmacy, Hue University, 06 Ngo Quyen Street, Hue, Vietnam;

${ }^{2}$ Becamex International Hospital, Thuan An City, Binh Duong, Vietnam;

${ }^{3}$ Hue University of Medicine and Pharmacy, Hue University, 06 Ngo Quyen Street, Hue, Vietnam;

${ }^{4}$ Department of OBGYN, Hue University of Medicine and Pharmacy, Hue University, 06 Ngo Quyen Street, Hue, Vietnam;

${ }^{5}$ Center for Reproductive Endocrinology and Infertility. Hue University of Medicine and Pharmacy, Hue University, 06 Ngo Quyen Street, Hue, Vietnam;

${ }^{6}$ Department of Microbiology, Institute of Biomedicine and Translational Medicine, University of Tartu, Tartu, 50411, Estonia;

${ }^{7}$ Institute of Clinical Medicine, Department of Obstetrics and Gynecology, University of Tartu, Tartu, 51014, Estonia;

${ }^{8}$ Division of Obstetrics and Gynecology, Department of Clinical Science, Intervention and Technology (CLINTEC), Karolinska Institutet, Stockholm, Sweden;

${ }^{9}$ Competence Centre on Health Technologies AS, Tartu, 50410, Estonia. 


\section{References}

1. Donders GG, Vereecken A, Bosmans E, Dekeersmaecker A, Salembier G, Spitz B. Definition of a type of abnormal vaginal flora that is distinct from bacterial vaginosis: aerobic vaginitis. BJOG: An International Journal of Obstetrics \& Gynaecology. 2002;109(1):34-43.

2. Kaambo E, Africa C, Chambuso R, Passmore J-AS. Vaginal microbiomes associated with aerobic vaginitis and bacterial vaginosis. Frontiers in public health. 2018;6:78.

3. Donders GG, Bellen G, Grinceviciene S, Ruban K, Vieira-Baptista P. Aerobic vaginitis: no longer a stranger. Research in Microbiology. 2017;168(9-10):845-858.

4. Hassan MF, Rund N, El-Tohamy O, Moussa M, Ali YZ, Moussa N, et al. Does aerobic vaginitis have adverse pregnancy outcomes? prospective observational study. Infectious diseases in obstetrics and gynecology. 2020;2020.

5. Hočevar K, Maver A, Vidmar Šimic M, Hodžić A, Haslberger A, Premru Seršen T, et al. Vaginal microbiome signature is associated with spontaneous preterm delivery. Frontiers in medicine. 2019;6:201.

6. Kaambo E, Africa CW. The threat of aerobic vaginitis to pregnancy and neonatal morbidity. African Journal of Reproductive Health. 2017;21(1):109-118.

7. Le TLL, Le MT. Lower genital tract infections in pregnant women over 35 weeks of gestation. Vietnam J Obstet Gynecol. 2016;14(3):44-48.

8. Oerlemans EF, Wuyts S, Bellen G, Wittouck S, De Boeck I, Ruban K, et al. The dwindling microbiota of aerobic vaginitis, an inflammatory state enriched in pathobionts with limited TLR stimulation. Diagnostics. 2020;10(11):879.

9. Schnarr J, Smaill F. Asymptomatic bacteriuria and symptomatic urinary tract infections in pregnancy. European journal of clinical investigation. 2008;38:50-57.

10. Lynch T, Peirano G, Lloyd T, Read R, Carter J, Chu A, et al. Molecular diagnosis of vaginitis: comparing quantitative PCR and microbiome profiling approaches to current microscopy scoring. Journal of clinical microbiology. 2019;57(9):e00300-19.

11. Wang C, Fan A, Li H, Yan Y, Qi W, Wang Y, et al. Vaginal bacterial profiles of aerobic vaginitis: a casecontrol study. Diagnostic microbiology and infectious disease. 2020;96(4):114981.

12. Mendling W. Vaginal microbiota. Microbiota of the human body. 2016:83-93.

13. Geng N, Wu W, Fan A, Han C, Wang C, Wang Y, et al. Analysis of the risk factors for aerobic vaginitis: a case-control study. Gynecologic and obstetric investigation. 2016;81(2):148-154.

14. Sonthalia S, Aggarwal P, Das S, Sharma P, Sharma R, Singh S. Aerobic vaginitis-An underdiagnosed cause of vaginal discharge-Narrative review. International journal of STD \& AIDS. 2020;31(11):1018-1027.

15. Krauss-Silva L, Almada-Horta A, Alves MB, Camacho KG, Moreira MEL, Braga A. Basic vaginal pH, bacterial vaginosis and aerobic vaginitis: prevalence in early pregnancy and risk of spontaneous 
preterm delivery, a prospective study in a low socioeconomic and multiethnic South American population. BMC Pregnancy and Childbirth. 2014;14(1):1-10.

16. Martín Saco G, García-Lechuz Moya JM. Update on vaginal infections: Aerobic vaginitis and other vaginal abnormalities. Prog obstet ginecol(Ed impr). 2019:72-78.

17. American College of Obstetricians, Practice GCoO. Prevention of Group B Streptococcal Early-Onset Disease in Newborns: ACOG Committee Opinion Summary, Number 797. Obstet Gynecol. 2020;135(2):489-492.

18. Gynecologists ACoOa. Prevention of Group B Streptococcal Early-Onset Disease in Newborns: ACOG Committee Opinion Summary, Number 782. Obstet Gynecol. 2019;134(1):1.

19. Verani JR, McGee L, Schrag SJ. Prevention of perinatal group B streptococcal disease: revised guidelines from CDC, 2010. 2010; 59(RR-10):1-36.

20. Nguyen TPL, Nguyen HB, Le VA, Nguyen TCA. Detection of group B Streptococcus (GBS) in pregnant women by SYBR Green real-time PCR. J Med Pharm. 2019;9(05):68.

21. Han C, Li H, Han L, Wang C, Yan Y, Qi W, et al. Aerobic vaginitis in late pregnancy and outcomes of pregnancy. European Journal of Clinical Microbiology \& Infectious Diseases. 2019;38(2):233-239.

22. Donders G, Greenhouse P, Donders F, Engel U, Paavonen J, Mendling W. Genital Tract GAS Infection ISIDOG Guidelines. J Clin Med. 2021 May 10;10(9):2043. 